\title{
ON THE BASIS NUMBER OF THE CORONA OF GRAPHS
}

\author{
MOHAMMAD SHAKHATREH AND AHMAD AL-RHAYYEL
}

Received 5 February 2005; Revised 19 June 2006; Accepted 22 June 2006

The basis number $b(G)$ of a graph $G$ is defined to be the least integer $k$ such that $G$ has a $k$ fold basis for its cycle space. In this note, we determine the basis number of the corona of graphs, in fact we prove that $b(v \circ T)=2$ for any tree and any vertex $v$ not in $T, b(v \circ H) \leq$ $b(H)+2$, where $H$ is any graph and $v$ is not a vertex of $H$, also we prove that if $G=G_{1}$ 。 $G_{2}$ is the corona of two graphs $G_{1}$ and $G_{2}$, then $b\left(G_{1}\right) \leq b(G) \leq \max \left\{b\left(G_{1}\right), b\left(G_{2}\right)+2\right\}$, moreover we prove that if $G$ is a Hamiltonian graph, then $b(v \circ G) \leq b(G)+1$, where $v$ is any vertex not in $G$, and finally we give a sequence of remarks which gives the basis number of the corona of some of special graphs.

Copyright (c) 2006 Hindawi Publishing Corporation. All rights reserved.

\section{Introduction}

In this note, we consider only finite, undirected, simple graphs. Our terminology and notation will be standard except as indicated. For undefined terms, see [7]. Let $G$ be a $(p, q)$ graph (i.e., $G$ has $p$ vertices and $q$ edges), and let $e_{1}, e_{2}, \ldots, e_{q}$ be an ordering of its edges. Then any subset $E$ of edges in $G$ corresponds to $(0,1)$-vector $\left(v_{1}, \ldots, v_{q}\right)$ with $v_{i}=1$ if $e_{i} \in E$ and $v_{i}=0$ if $e_{i} \notin E$. The vectors form a $q$-dimensional vector space over the field of two elements $Z_{2}$ and is denoted by $\left(Z_{2}\right)^{q}$. The vectors in $\left(Z_{2}\right)^{q}$ which correspond to the cycles in $G$ generate a subspace called the cycle space of $G$ and is denoted by $C(G)$, we will say that the cycles themselves, instead of saying the vectors corresponding to the cycles, generate $C(G)$. It is well known (see [7, page 39]) that if $G$ is a $(p, q)$ graph with $k$ components, then $\operatorname{dim} C(G)=\gamma(G)=q-p+k$, where $\gamma(G)$ is the cyclomatic number of $G$. A basis for $C(G)$ is called a $k$-fold basis if each edge of $G$ occurs in at most $k$ of the cycles in the basis. The basis number of $G$ denoted by $b(G)$ is the smallest integer $k$ such that $C(G)$ has a $k$-fold basis. The corona (see [7, page 167]) of two graphs $G_{1}$ and $G_{2}$, denoted by $G_{1} \circ G_{2}$, is defined to be the graph $G$ obtained by taking one copy of $G_{1}$ (which has $p_{1}$ vertices) and $p_{1}$ copies of $G_{2}$, and then joining the vertex of $G_{1}$ to every vertex in the $i$ th copy of $G_{2}$. If $G_{1}$ is a $\left(p_{1}, q_{1}\right)$ graph and $G_{2}$ is a $\left(p_{2}, q_{2}\right)$ graph, then it follows from the definition of the corona that $G_{1} \circ G_{2}$ has $p_{1}\left(1+p_{2}\right)$ vertices and $q_{1}+p_{1} q_{2}+p_{1} p_{2}$ edges 
(see [7, page 168]). It is clear that if $G_{1}$ is connected, then $G_{1} \circ G_{2}$ is connected, and in general $G_{1} \circ G_{2}$ is not isomorphic to $G_{2} \circ G_{1}$.

In the rest of this note, $P_{n}, C_{n}, S_{n}$, and $W_{n}$ stand for the path, the cycle, the star, and the wheel of $n$ vertices. A theta graph $\theta_{n}$ is defined to be a cycle $C_{n}$ with $n$ vertices, respectively, to which we add a new edge that joins two nonadjacent vertices of $C_{n}$.

MacLane [8] proved that a graph $G$ is planar if and only if $b(G) \leq 2$. Schmeichel [9] proved that for $n \geq 5, b\left(K_{n}\right)=3$ and for $m, n \geq 5, b\left(K_{m, n}\right)=4$ except for $K_{6,10}, K_{5, n}$, and $K_{6, n}$, where $n=5,6,7$, and 8. Banks and Schmeichel [6] proved that for $n \geq 7, b\left(Q_{n}\right)=7$, where $Q_{n}$ is the $n$-cube. Ali [1] proved that $b\left(K_{n, n, \ldots, n}\right) \leq 9, b\left(K_{n, n, n}\right)=3$ for all $n \geq 3$, and $b\left(K_{l, m, n}\right) \leq 4$. Moreover, Ali [2] proved that $b\left(C_{m} \wedge P_{n}\right) \leq 2$, and $b\left(C_{m} \wedge C_{n}\right)=3$. Al-Rhayyel [4] proved that $b\left(P_{2} \times \theta_{n}\right)=2$ and $b\left(\theta_{n} \times \theta_{m}\right)=3$ for all $n, m \geq 4$. Al-Rhayyel [5] proved that $b\left(P_{2} \wedge W_{n}\right)=2$ and $b\left(P_{m} \wedge W_{n}\right)=3$ for all $m \geq 3, n \geq 4$, and $n$ is even where $\wedge$ and $\times$ are the direct and the cartesian products of graphs, respectively. Next we restate [3, Theorem 2.3].

Theorem 1.1. Let $G^{\prime}$ be a graph obtained from $G$ by deleting an edge e of at most 2-fold in $a$ basis $B$ for $C(G)$. Then $b(G)-1 \leq b\left(G^{\prime}\right) \leq b(G)$.

The purpose of this note is to investigate the basis number of the corona of graphs, in fact we prove that for any two graphs $G_{1}$ and $G_{2}$, if $G=G_{1} \circ G_{2}$, then $b\left(G_{1}\right) \leq b(G) \leq$ $\max \left\{b\left(G_{1}\right), b\left(G_{2}\right)+2\right\}$ and we give the exact basis number of the corona of some special graphs.

\section{Main results}

This section is devoted for proving the main results of this note, and this is done by writing a sequence of theorems and remarks.

Remark 2.1. We note that if $v$ is not a vertex of $G$, then $b(v \circ G)=2$, where $G$ is any one of the following graphs: $P_{n}, C_{n}$ or $S_{n}$ and $b(v \circ G)=3$ if $G$ is either $W_{n}$ or $K_{n}(n \geq 4)$.

Lemma 2.2. Let $T$ be a tree with $p$ vertices $(p \geq 3)$ if $v$ is any point which is not a vertex of $T$, and if $G=v \circ T$, then $b(G)=2$, and hence $G$ is planar.

Proof. Assume that $G$ is not planar. Then, by Kuratowski's theorem, $G$ contains a subdivision of $K_{5}$ or $K_{3,3}$. Then $G-x$ cannot be acyclic graph for any $x \in V(G)$, while $G-v$ is a tree. This is a contradiction, and hence $G$ is planar. Therefore, $b(G) \leq 2$. If $b(G)=1$, then $G$ has a 1 -fold basis, which implies that $\operatorname{dim} C(G) \leq|E(G)| / 3$ since each cycle contains at least three edges. Since $|E(G)|=2 p-1$ and $\operatorname{dim} C(G)=p-1$, we have $p-1 \leq$ $(2 p-1) / 3$, which implies that $p \leq 2$. This is a contradiction. Therefore, $b(G)=2$.

Lemma 2.3. Let $H$ be any connected $(p, q)$ graph and let $v$ be any vertex which is not a vertex of $H$. If $G=v \circ H$, then $b(G) \leq b(H)+2$.

Proof. Let $u_{1}, u_{2}, \ldots, u_{p}$ be the vertices of $H$. Since $\operatorname{dim} C(G)=q$ and $\operatorname{dim} C(H)=q-$ $p+1, \operatorname{dim} C(G)-\operatorname{dim} C(H)=p-1$. Let $T$ be a spanning tree of $H$. Then $b(v \circ T)=2$, $\operatorname{dim} C(v \circ T)=p-1$, and each cycle in $v \circ T$ must contain an edge of the form $v u_{i}$ for some $i \in\{1,2, \ldots, p\}$. Thus the cycles in $v \circ T$ are independent from the cycles in $H$. Let $B_{1}$ be a $b(H)$-fold basis for $C(H)$, and let $B_{2}$ be a 2 -fold basis for $v \circ T$. Then clearly 
$B=B_{1} \cup B_{2}$ is an independent set of cycles with $|B|=\operatorname{dim} C(G)$, hence $B$ is a basis for $C(G)$. Note that if $e$ is an edge of $G$, then either $e$ is an edge of $H$ or $e=v u_{i}$ for some $i \in\{1,2, \ldots, p\}$. If $e=v u_{i}$, then $f_{B}(e) \leq f_{B_{2}}(e) \leq 2$, and if $e$ is an edge of $H$, then clearly $f_{B}(e) \leq b(H)+2$. Thus, $b(G) \leq b(H)+2$.

Theorem 2.4. Let $G_{1}$ and $G_{2}$ be two connected graphs. If $G=G_{1} \circ G_{2}$, then $b\left(G_{1}\right) \leq b(G) \leq$ $\max \left\{b\left(G_{1}\right), b\left(G_{2}\right)+2\right\}$.

Proof. Clearly $b\left(G_{1}\right) \leq b(G)$. Let $v_{1}, \ldots, v_{n}$ be the vertices of $G_{1}$ and let $H_{k}=v_{k} \circ G_{2}$ and let $B_{k}$ be the basis of $H_{k}$, for each $k=1, \ldots, n$. Clearly $E\left(H_{i}\right) \cap E\left(H_{j}\right)=\phi$, for all $i \neq j i, j \in$ $\{1, \ldots, n\}$. Therefore, $\bigcup_{k=1}^{n} B_{k}$ is linearly independent. Let $B=\left(\bigcup_{k=1}^{n} B_{k}\right) \cup B\left(G_{1}\right)$, where $B\left(G_{1}\right)$ is a $b\left(G_{1}\right)$-fold basis for $G_{1}$. Since $E\left(G_{1}\right) \cap E\left(\left(\bigcup_{k=1}^{n} H_{k}\right)\right)=\phi$, as a result $B$ is linearly independent. Since $|E(B)|=\operatorname{dim} C(G), B$ is a basis of $C(G)$. By Lemma 2.3, $b\left(H_{k}\right) \leq$ $b\left(G_{2}\right)+2$ for each $k \in\{1, \ldots, n\}$. Therefore, $b\left(G_{1}\right) \leq b(G) \leq \max \left\{b\left(G_{1}\right), b\left(G_{2}\right)+2\right\}$.

Lemma 2.5. Let $H$ be a Hamiltonian graph, and let $v$ be any point which is not a vertex of $H$. If $G=v \circ H$, then $b(G) \leq b(H)+1$.

Proof. Let $C=u_{1} u_{2}, \ldots, u_{n} u_{1}$ be a spanning cycle of $H$, then $G$ is obtained from $H$ by joining every vertex $v_{i}$ of $H$ to the vertex $v$. Let $B=\left\{v u_{i} u_{i+1}: i=1,2, \ldots, n-1\right\} \cup \mathrm{B}(H)$, where $B(H)$ is a $b(H)$-fold basis of $C(H)$. Then, clearly that $B$ is a basis of $C(G)$ and given any edge of $H$, then it occurs in at most one of these cycles, hence $b(G) \leq b(H)+1$.

Corollary 2.6. If $G_{1}$ and $G_{2}$ are two graphs such that $b\left(G_{1}\right) \geq b\left(G_{2}\right)+2$, then $b\left(G_{1}\right.$ 。 $\left.G_{2}\right)=b\left(G_{1}\right)$. Moreover, if $G_{2}$ is Hamiltonian, and $b\left(G_{1}\right) \geq b\left(G_{2}\right)+1$, then $b\left(G_{1} \circ G_{2}\right)=$ $b\left(G_{1}\right)$.

\section{References}

[1] A. A. Ali, The basis number of complete multipartite graphs, Ars Combinatoria 28 (1989), 41-49.

[2] $155-163$.

[3] A. A. Ali and S. AL-Sardary, On the basis number of graph, Dirasat 14 (1987), no. 1, 43-51.

[4] A. Al-Rhayyel, On the basis number of the Cartesian product of theta graphs, Mathematica Japonica 41 (1995), no. 2, 327-330.

[5] - On the basis of the direct product of paths and wheels, International Journal of Mathematics and Mathematical Sciences 19 (1996), no. 2, 411-414.

[6] J. A. Banks and E. F. Schmeichel, The basis number of the n-cube, Journal of Combinatorial Theory. Series B 33 (1982), no. 2, 95-100.

[7] F. Harary, Graph Theory, 2nd ed., Addison-Wesley, Massachusetts, 1971.

[8] S. MacLane, A combinatorial condition for planar graphs, Fundamenta Mathematicae 28 (1937), $22-32$.

[9] E. F. Schmeichel, The basis number of a graph, Journal of Combinatorial Theory. Series B 30 (1981), no. 2, 123-129.

Mohammad Shakhatreh: Department of Mathematics, Yarmouk University, Irbid 211-63, Jordan E-mail address: mali@yu.edu.jo

Ahmad Al-Rhayyel: Department of Mathematics, Yarmouk University, Irbid 211-63, Jordan E-mail address: alrahayyel@yahoo.com 


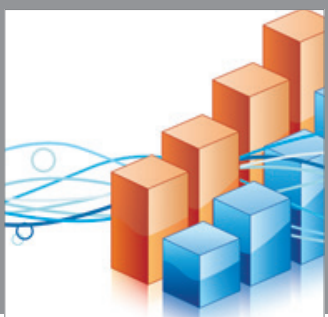

Advances in

Operations Research

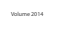

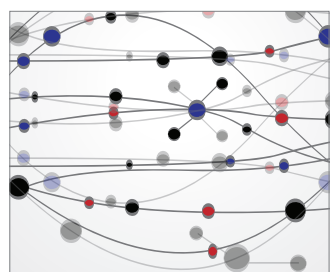

\section{The Scientific} World Journal
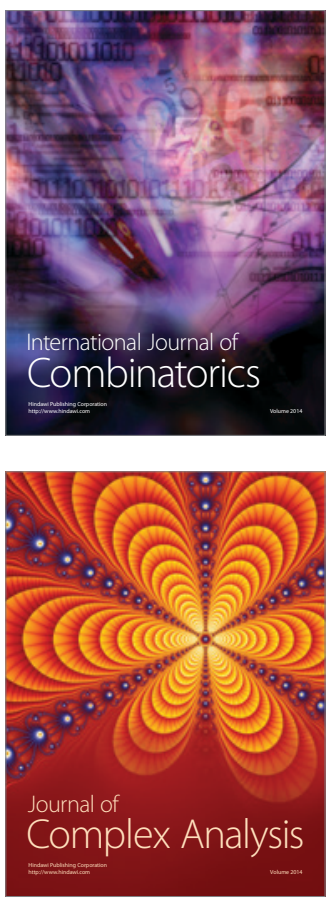

International Journal of

Mathematics and

Mathematical

Sciences
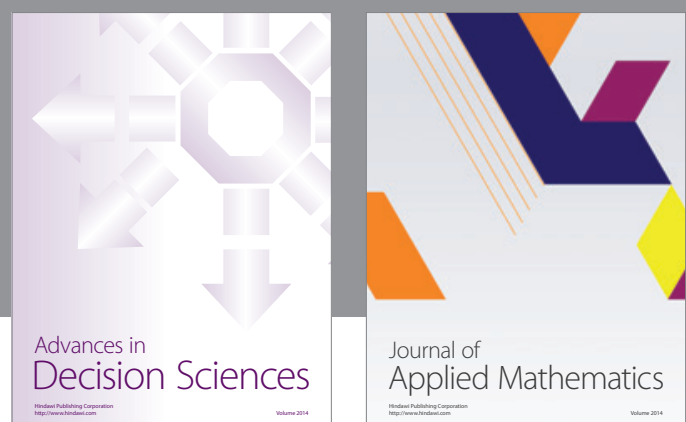

Journal of

Applied Mathematics
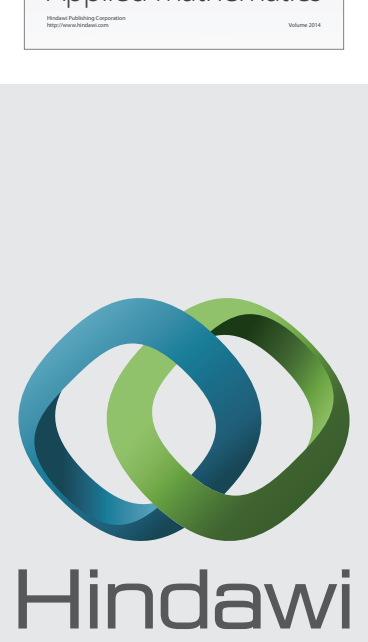

Submit your manuscripts at http://www.hindawi.com
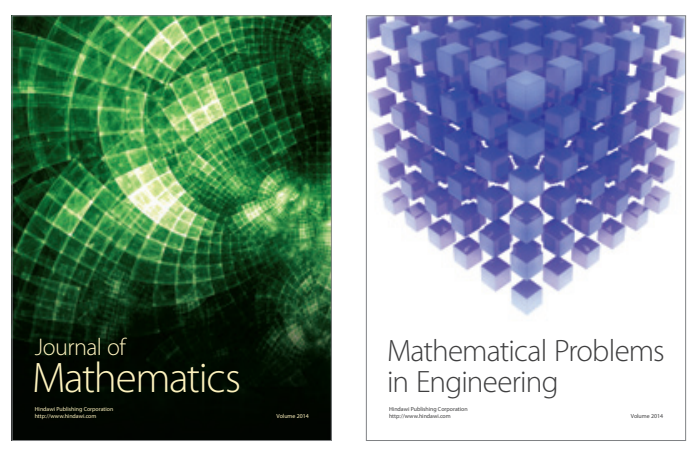

Mathematical Problems in Engineering
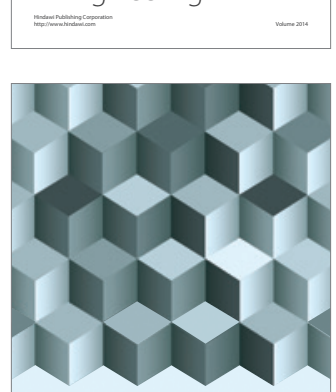

Journal of

Function Spaces
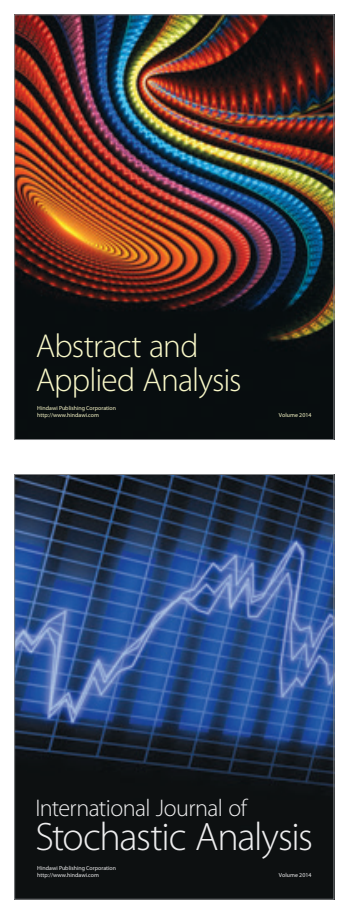

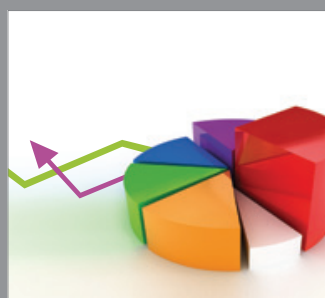

ournal of

Probability and Statistics

Promensencen
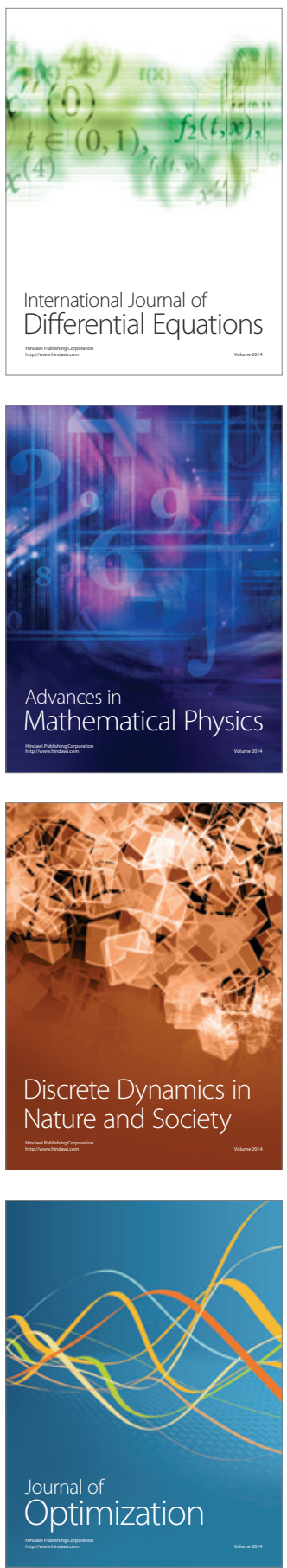